\title{
Interpersonal attraction, topic importance, and proportion of item agreements
}

\author{
PAUL G. BANIKIOTES \\ University of Notre Dame, Notre Dame, Ind. 46556
}

The program of research initiated by Byrne (1961) has repeatedly demonstrated that the proportion of item agreements between an individual and a hypothetical stranger represented by a test protocol is the most critical variable determining interpersonal attraction. The importance of the topic or item has only received occasional support as a determining factor. The purpose of this investigation was to contrast the effect of topic importance with the effect of proportion of item agreements as they relate to interpersonal attraction. The results of this study indicate that the effect of topic importance may be greater than had previously been thought.

The proportion of item agreements between an individual and a hypothetical stranger represented by a test protocol has repeatedly been found to be the most critical variable determining interpersonal attraction (Byrne, 1961; Byrne, 1964; Byrne \& Clore, 1966; Byrne, Clore, \& Griffitt. 1967). Inconsistent results have been reported with regard to the effect of topic importance on attraction. Byrne $\&$ Nelson $(1964,1965)$ found that only proportion of similar attitudes had a significant effect on attraction, while topic importance did not. Findings from two studies (Byrne, London, \& Griffitt, 1968; Clore \& Baldridge, 1968) indicated that importance of attitudinal topics is a relevant variable under certain conditions, that is, if the items are heterogeneous and if the similarity level is at an intermediate point between .00 and 1.00. These conditions, however, are those most likely to exist in a real life situation. Banikiotes (1970), in contrasting interpersonal attraction methodology (Byrne, 1961) with sociometric ratings, found that topic importance was critical in determining attraction in real-life situations.

It was the purpose of this investigation to contrast the effect of topic importance with the effect of proportion of item agreements as they relate to interpersonal attraction in a simulated laboratory situation. It was hypothesized that topic importance would play a greater role in determining attraction than would pruportion of item agreements.

\section{METHOD \\ Subjects}

The Ss were students in a graduate course in education. The 28 male and female Ss ranged from 22 to 32 years of age.

\section{Instrumentation}

A group of 12 items referred to as the attitude survey (A-S) was employed.
The items of the A-S were presented as simple six-choice scales. These are the same items which have been used in previous simulated laboratory studies employing the interpersonal attraction methodology. Nelson $(1969)^{1}$ developed this particular group of items as one of two parallel forms to measure attitudes. The items are heterogeneous with respect to topic importance.

The Interpersonal Judgment Scale (IJS) (Byrne, 1961) was used to evaluate the Ss' responses to the hypothetical individuals represented by test data.

\section{Procedure}

The study involved two testing sessions. In the first session, the A-S was administered to each $\mathrm{S}$. In addition, Ss were asked to rate each of the 12 items on a 5-point scale according to its importance to them.

In the second session, each $S$ was presented with two A-S test response protocols which purportedly represented individuals of the same sex and age who were not known. The test protocols were, in fact, contrived such that one was in agreement with the individual's responses on 9 of the 12 items but disagreed on the most important items, while the other was in agreement with the individual's responses on 6 of the 12 items but agreed on the most important items. After reading each test protocol, each $S$ rated the hypothetical stranger on the IJS. In addition, the $S$ was asked to choose which of the two strangers he liked better.

\section{Analysis}

A $t$ test was used to determine if the IJS rating for the two hypothetical stranger categories differed. A chi-square analysis was computed to compare the expected distribution of better-liked choices with the observed distribution. The .01 level was held critical for all tests made.
RESULTS AND DISCUSSION

A significant difference was found between IJS ratings of the two categories of the A-S protocols of hypothetical strangers $(t=3.03)$. The stranger who agreed on only $50 \%$ of the items but agreed on the most important ones was rated higher than the stranger who agreed on $75 \%$ of the items but disagreed on the most important ones. Also, the observed choices of better-liked individuals differed significantly from expectation $\left(x^{2}=9.14\right)$. The difference was in the same direction as for the IJS ratings.

As hypothesized, topic importance, when contrasted with proportion of item agreements, was found to be of greater relevance in determining interpersonal attraction. The critical nature of topic importance seems to hold true in simulated laboratory situations as well as in real life. Results from previous simulated laboratory experiments have indicated that topic importance is relevant only under certain specific conditions, that is, heterogeneous items and similarity level between .00 and 1.00 . It is these very conditions, however, which are the ones most likely to exist in real-life situations. It is unrealistic to generalize from a laboratory situation to a real-life situation if a group of homogeneous items has been used and proportion of item agreements is only $0 \%$ or $100 \%$.

The necessity for continuity between the study of attraction in the laboratory and its manifestation under field conditions has been well documented (Byrne, Ervin, \& Lamberth, 1970). The closer laboratory conditions are to those in the real world, the greater are the chances that the results will generalize. It may be that much time has been spent in the study of attitudinal topics which have little relevance in determining interpersonal attraction in real-life relationships. It appears from the findings of this and other studies that more attention should be focused on the topic importance of the attitudes employed.

\section{REFERENCES}

BANIKIOTES, P. G. Interpersonal attraction in simulated and real interactions. Unpublished doctoral dissertation, Purdue University, 1970.

BYRNE, D. Interpersonal attraction and attitude similarity. Journal of Abnormal \& Social Psychology, 1961, 62, 713-715.

BYRNE, D. Repression-sensitization as a dimension of personality. In B. A. Maher (Ed.). Progress in experimental personality research. Vol. 1. New York: Academic Press, 1964. Pp. 169-220.

BYRNE, D., \& CLORE, G. L., JR. Predicting interpersonal attraction toward strangers presented in three different stimulus modes. Psychonomic Science, 1966, 4 239-240.

BYRNE, D., CLORE, G. L., JR. \& GRIFFITT, W. Response discrepancy versus attitude similarity as determinants of at traction. Psychonomic Science, 1967. 7, 397-398. 
BYRNE, D. ERVIN, C R., \& LAMBERTH $J$. Continuity between the experimental study of attraction and real-life computer dating. Journal of Personality \& Social Psychology, 1970, 16, 157-165.

BYRNE, D., LONDON, O., GRIFFITT, $w$ The effect of topic importance and attitude similarity-dissimilarity on attraction in an intrastranger design. Psychonomic Science, 1968, 11, 303-304.

BYRNE, D., \& NELSON, D. Attraction as a function of attitude similaritydissimilarity: The effect of topic importance. Psychonomic Science, 1964, 1. 93-94.
BYRNE, D.. \& NELSON, D. Attraction as a linear function of proportion of positive reinforcements. Journal of Personality \& Social Psychology , 1965, 1, 659-663.

CLORE, G. L. \& BALDRIDGE, B. Interpersonal attraction: The role of agreement and topic interest. Journal of Personality \& Social Psychology. 1968,9, 340-346.

\section{NOTE}

1. Nelson, D. Personal communication, October 1969.

\section{Acoustic interference}

\section{with redundant elements*}

\author{
ROBERT A. SMALLWOOD and L. JAMES TROMATER \\ University of Richmond, Richmond, Va. 23173
}

The acoustic similarity and neutrality of letters was varied as response prefixes and stimulus suffixes and as members of a memory series in an immediate memory task. Each of 32 Ss vocalized 160 visually presented lists composed of randomly selected letters from an acoustically similar subset (BCDGPTVZ) and a neutral subset (HJLNRXQY), followed by ordered vocal recall. Half the Ss received each letter from the acoustically similar subset five times as a redundant element (additional letter) in a response prefix, a stimulus suffix, and a modified stimulus suffix condition, while the other half correspondingly received letters from the neutral subset. Predictions based on retroactive interference explanations of the similarity of the redundant letters to members of a memory series failed to be supported. In addition, the extent to which a stimulus suffix interferes with the final items in a series is independent of the spatial separation of that suffix.

Investigations of redundant elements in an immediate memory task involving the occurrence of a predictable element between the visual or aural presentation of a stimulus string and the beginning of recall have

* This research was supported, in part, by a grant from the University of Richmond. Richmond, Va. response prefix. Althougi therr effects upon performance are of comparable magnitude, their seemingly equivalent nature is negated by theic differential effects on the serial position function. The stimulus suffix has been found to selectively affect the recall of the final items in a list, while the response prefix affects the recall of all the items to the same extent (Crowder, 1967; Morton, 1968).

Crowder \& Morton (1969) have proposed a subsystem of human memory called precategorical acoustic storage (PAS), which accounts for the serial position effects. Their model of information flow postulates a precategorical holding mechanism receptive to information only from Ss' ears. The material within PAS is subject to both overwriting and decay, with a maximum useful life of about 2 sec. The interpretation placed on the stimulus suffix position-specific effect is that hearing the redundant element displaces PAS traces $S$ would otherwise be able to use during rehearsal. Since, at the time the suffix is presented, there are no PAS traces still existing for the earlier serial positions, the effect is selective in impairing performance for the late positions.

While PAS accounts for the position-specific effects of the stimulus suffix, the effects of the response prefix are not directly suggested by the data which Crowder and Morton used to substantiate their information-flow model. They do, however, propose that the nonselective overall effect on errors with a response prefix is due to articulatory interference, since articulatory coding is a perceptual consequence for all elements in the list. Crowder (1969) has noted that the response prefix may be said, on operational grounds, to exemplify retroactive inhibition (RI) by virtue of its location between input and output. If the response prefix effect can be accounted for by basic laws of RI, then the similarity of the prefix element to the members of the memory series should be directly related to the size of the prefix effect.

Crowder (1967, Experiment 3) rejected the $R I$ interpretation of the prefix effect, but in a more recent study (1969) has confirmed an RI account of the prefix effect by showing that when the dominant phoneme in a memory list was "e," a larger decrement was obtained when the prefix was the letter " $v$ " than when it was "k." The present experiment sought to test further the hypothesis that the prefix effect is directly associated with the degree of phonic similarity between the memory series and the prefix element, by representing a more complete range of 